\title{
INTRODUCING A NOVEL METHOD TO SOLVE SHORTEST PATH PROBLEMS BASED ON STRUCTURE OF NETWORK USING GENETIC ALGORITHM
}

\author{
S. Behzadi ${ }^{1 *}$, M. Kolbadinejad ${ }^{2}$ \\ ${ }^{1}$ Faculty of Civil Engineering, Shahid Rajaee Teacher Training University, Tehran, Iran, behzadi.saeed@ gmail.com \\ ${ }^{2}$ The Ministry of Interior of the Islamic Republic of Iran, fereshtek630@ gmail.com
}

Commission VI, WG VI/4

KEY WORDS: Genetic Algorithm, Shortest path, Network design, multistage process planning, directed acyclic network

\begin{abstract}
:
The shortest path problem is widely applied in transportation, communication and computer networks. It addresses the challenges of determining a path with minimum distance, time or cost from a source to the destination. Network analysis provides strong decision support for users in searching shortest path. A lot of algorithms are designed for solving shortest path but most of them did not considered the conditions of the networks. Genetic Algorithm is a kind of Algorithm that has a lot of efficiency and it can be used for solving many kinds of problems. It also can be used based on the condition of the problems.

In this paper, Genetic Algorithm is used for solving shortest path in multistage process planning (MPP) problem. New mutations as well as crossover parameters are defined for each network based on the conditions of them. The results of our experimental demonstrate the effectiveness of the models.
\end{abstract}

\section{INTRODUCTION}

\subsection{Introduction}

Genetic algorithms (GAs) are one of the most powerful and optimization techniques based on principles from evolution theory (Li, Sun, Tseng, \& Li, 2019; Zero, Bersani, Paolucci, \& Sacile, 2019). Over the past few years, the GAs community has turned much of its attention toward the optimization of network design problem (Ergenç, Eksert, \& Onur, 2019; Hanh, Binh, Hoai, \& Palaniswami, 2019; Kaur, Singh, \& Kaur, 2019). In this paper, we summarized recent research works on network design problem by using genetic algorithms (GAs), including multistage process planning (MPP) problem, a directed acyclic network problem a local network and a real network then some genetic algorithms are introduced for solving these types of optimization problems arising in the field of network planning these genetic algorithms are applied to find shortest path in network.

\subsection{Genetic Algorithm}

Genetic algorithms are inspired by Darwin's theory about evolution. Solution to a problem solved by genetic algorithms is evolved. The genetic algorithm is a method for solving optimization problems that is based on natural selection (Chenini, Aissat, \& Vilcot, 2019; Cheong \& Koh, 2019; Wang, Duan, \& Yang, 2018; Zhao \& Liu, 2018), the process that drives biological evolution. The genetic algorithm repeatedly modifies a population of individual solutions (Pérez-Galarce, Candia-Véjar, Astudillo, \& Bardeen, 2018). At each step, the genetic algorithm selects individuals at random from the current population to be parents and uses them produce the children for the next generation (Peng et al., 2018). Over successive generations, the population "evolves" toward an optimal solution (Chen, Tong, Lu, \& Wang, 2018; Flick, 2018; Mousavi \& Behzadi, 2019; Nasr, El-Bahnasawy, Attiya, \& El-Sayed, 2018).

\section{MULTISTAGE PROCESS PLANNING}

Multistage process planning (MPP) problem is abundant in manufacturing system. It provides a detailed description of manufacturing capability and requirements for transforming a raw stock of materials into a completed product through multistage process. The MPP problem is to find the optimal process planning among all possible alternative given certain criteria such as minimum cost, minimum time, maximum quality or under multiple of these criteria (Ahmadi, Süer, \& Al-Ogaili, 2018; Bäck, Fogel, \& Michalewicz, 2018). This problem can be considered in transportation network as an exclusive network that an object is flowed in an optimal path in this network. Fig. 1 shows a simple example of the MPP problem by means of network flows.

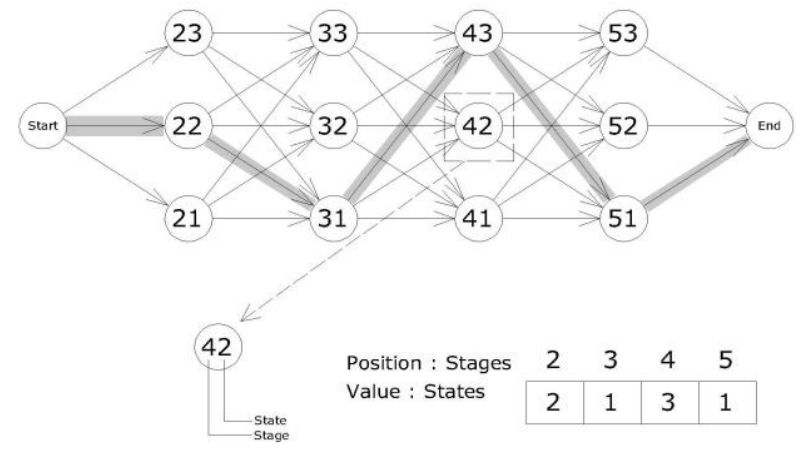

Fig. 1. Flow network for a simple MPP problem

\footnotetext{
* Corresponding author
} 


\subsection{Coding}

In this case, there exists an ordered network which has some steps. Each step contains some nodes as the number of these nodes at each step is equal with each other and it is constant. Permutation encoding can be used in this problem. The position of a gene is used to indicate the stage and the value of the gene is used to indicate a state at that stage. Because the state for the first stage is always fixed, so we don't need to encode this state in a chromosome. It means that for a given problem with $n$ stages, we have the length of an encoding n-1 (Tong, Wu, Jiang, Yu, \& Rao, 2017; Xiao, Xie, Kulturel-Konak, \& Konak, 2017). Each point in the map is given a unique integer value index like $[\mathrm{MN}]$, where, $\mathrm{M}$ is the number of stage in the map and $\mathrm{N}$ is the number of state in the stage (Behzadi, Alesheikh, \& Poorazizi, 2008).

For example in instance given in Fig. 1, the final rout consists 22, 31, 43 and 51. The first section of these numbers corresponds to the stages of these numbers as shown [2 34 ] and the second section of them corresponds to the state of them in their stage that can be shown $\left[\begin{array}{llll}2 & 1 & 3 & 1\end{array}\right]$.

\subsection{Initial Population}

Initial population is generated randomly. As considered, the length of an encoding chromosome depends on the number of stages. Each gene of this chromosome is defined by choosing randomly one of the nodes in corresponding stage.

\subsection{Fitness Function}

The fitness function of each individual in this problem is the sum of the weights of the connected lines between two adjacency nodes (Deo, 2017; Dib, Manier, Moalic, \& Caminada, 2017). For example $(i, j, k)=C_{i j}+C_{j k}$ is one individual that the length of it is 3 and it represents the connection between $\mathrm{i}, \mathrm{j}$ and $\mathrm{j}, \mathrm{k}$ as $C_{i j}+C_{j k}$ is its fitness function.

\subsection{Fitness Scaling}

Fitness scaling converts the raw fitness scores that are returned by the fitness function to values in a range that is suitable for the selection function. The selection function uses the scaled fitness values to select the parents of the next generation. The selection function assigns a higher probability of selection to individuals with higher scaled values (Bakirtzis \& Kazarlis, 2016; Emary, Zawbaa, \& Hassanien, 2016).

After creating the initial population, fitness values for each individual are calculated. In this research, proportional scaling is used to make the scaled value of an individual proportional to its raw fitness score.

\subsection{Genetic Operator}

Genetic operators mimic the process of heredity of genes to generate new offspring at each generation and play a very important role in genetic algorithm (Askarzadeh, 2016). In this problem only mutation operator is used to produce offspring. It works with the following three major steps (Malawski, Juve, Deelman, \& Nabrzyski, 2015; Yu, Li, Jia, Zhang, \& Wang, 2015):
1. Determine the mutated gene randomly for a given chromosome.

2. Create a set of neighbour by replacing the mutated gene with all its possible states.

3. Select the best one from the neighbours as offspring.

Fig. 2 shows as an example of the neighbourhood search-based mutation method.

\begin{tabular}{|c|c|c|c|c|c|c|}
\hline \multicolumn{6}{|c|}{$\begin{array}{c}\text { Mutated gene } \\
\downarrow\end{array}$} & \multirow[b]{2}{*}{ Parent } \\
\hline Start & 2 & 1 & 3 & 1 & End & \\
\hline Start & 2 & 2 & 3 & 1 & End & \multirow[t]{4}{*}{ Neighbor } \\
\hline Start & 2 & 3 & 3 & 1 & End & \\
\hline & & . & & & & \\
\hline Start & 2 & a & 3 & 1 & End & \\
\hline
\end{tabular}

Fig. 2. Illustration of mutation with neighbourhood search.

\subsection{Experiments}

To evaluate the performance of the outlined method, we performed experiments using an instance of the PMM problem with 17 stages and 98 nodes we generated. One attribute was defined on each arc. This attributes were considered as the weight of the arcs. The objective was to minimize the total weight of path. The result of this experiment is shown in Fig. 3.

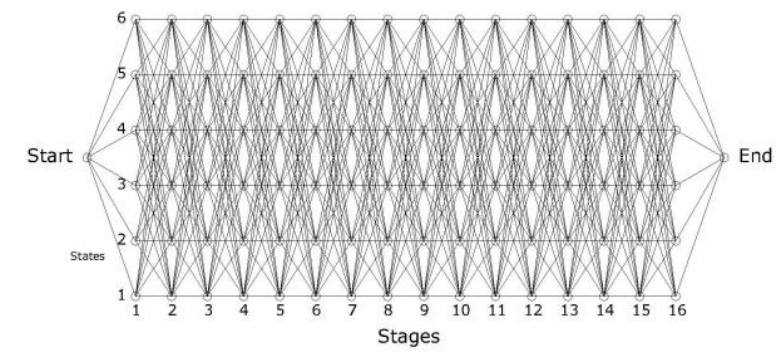

Fig. 3. The solution of the multiple objective MPP problem.

\section{CONCLUSIONS}

The main advantage of genetic algorithms is their flexibility. One thing that is striking about genetic algorithms is the richness of this form of computation. What may seem like simple changes in the algorithm often result in surprising kinds of emergent behaviour. By changing the parameters and operators of this algorithm, we can find the best and fast solution for the problem. Recent theoretical advances have also improved the understand ability of genetic algorithms and have opened the door to using more advanced analytical methods.

The research is to propose some methods to solve the shortest path problem using genetic algorithms in multistage process planning (MPP). The solution aims to achieve an increased number of successful and valid convergence using evolutionary computing techniques.

\section{REFERENCES}

Ahmadi, E., Süer, G. A., \& Al-Ogaili, F. (2018). Solving Stochastic Shortest Distance Path Problem by Using 
Genetic Algorithms. Procedia Computer Science, $140,79-86$.

Askarzadeh, A. (2016). A novel metaheuristic method for solving constrained engineering optimization problems: crow search algorithm. Computers \& Structures, 169, 1-12.

Bäck, T., Fogel, D. B., \& Michalewicz, Z. (2018). Evolutionary computation 1: Basic algorithms and operators: CRC press.

Bakirtzis, A., \& Kazarlis, S. (2016). Genetic algorithms. Advanced Solutions in Power Systems: HVDC, FACTS, and Artificial Intelligence: HVDC, FACTS, and Artificial Intelligence, 845-902.

Behzadi, S., Alesheikh, A. A., \& Poorazizi, E. (2008). Developing a genetic algorithm to solve shortest path problem on a raster data model. Journal of Applied Sciences, 8(18), 3289-3293.

Chen, P., Tong, R., Lu, G., \& Wang, Y. (2018). The $\alpha$-reliable path problem in stochastic road networks with link correlations: A moment-matching-based path finding algorithm. Expert Systems with Applications, 110, 2032.

Chenini, L., Aissat, A., \& Vilcot, J. (2019). Optimization of inter-subband absorption of InGaAsSb/GaAs quantum wells structure. Superlattices and Microstructures.

Cheong, K. H., \& Koh, J. M. (2019). A Hybrid GeneticLevenberg Marquardt Algorithm for Automated Spectrometer Design Optimization. Ultramicroscopy.

Deo, N. (2017). Graph theory with applications to engineering and computer science: Courier Dover Publications.

Dib, O., Manier, M.-A., Moalic, L., \& Caminada, A. (2017). Combining VNS with genetic algorithm to solve the one-to-one routing issue in road networks. Computers \& Operations Research, 78, 420-430.

Emary, E., Zawbaa, H. M., \& Hassanien, A. E. (2016). Binary grey wolf optimization approaches for feature selection. Neurocomputing, 172, 371-381.

Ergenç, D., Eksert, L., \& Onur, E. (2019). Dependability-based Clustering in Mobile Ad-Hoc Networks. Ad Hoc Networks, 101926.

Flick, U. (2018). An introduction to qualitative research: Sage Publications Limited.

Hanh, N. T., Binh, H. T. T., Hoai, N. X., \& Palaniswami, M. S. (2019). An efficient genetic algorithm for maximizing area coverage in wireless sensor networks. Information Sciences, 488, 58-75.

Kaur, P., Singh, G., \& Kaur, P. (2019). An intelligent validation system for diagnostic and prognosis of ultrasound fetal growth analysis using Neuro-Fuzzy based on genetic algorithm. Egyptian Informatics Journal, 20(1), 55-87.

Li, L.-L., Sun, J., Tseng, M.-L., \& Li, Z.-G. (2019). Extreme learning machine optimized by whale optimization algorithm using Insulated Gate Bipolar Transistor module aging degree evaluation. Expert Systems with Applications.

Malawski, M., Juve, G., Deelman, E., \& Nabrzyski, J. (2015). Algorithms for cost-and deadline-constrained provisioning for scientific workflow ensembles in IaaS clouds. Future Generation Computer Systems, 48, $1-18$.

Mousavi, Z., \& Behzadi, S. (2019). Geo-Portal Implementation with a Combined Approach of AHP and SWOT. International Journal of Natural Sciences Research, 7(1), 22-31. Retrieved from http://www.pakinsight.com/archive/63/01-2019/1
Nasr, A. A., El-Bahnasawy, N. A., Attiya, G., \& El-Sayed, A. (2018). A new online scheduling approach for enhancing QOS in cloud. Future Computing and Informatics Journal, 3(2), 424-435.

Peng, H., Li, J., He, Y., Liu, Y., Bao, M., Wang, L., . . Yang, Q. (2018). Large-scale hierarchical text classification with recursively regularized deep graph-cnn. Paper presented at the Proceedings of the 2018 World Wide Web Conference.

Pérez-Galarce, F., Candia-Véjar, A., Astudillo, C., \& Bardeen, M. (2018). Algorithms for the Minmax Regret Path problem with interval data. Information Sciences, 462, 218-241.

Tong, J., Wu, C., Jiang, H., Yu, Y., \& Rao, X. (2017). Optimizing the path of seedling low-density transplanting by using greedy genetic algorithm. Computers and Electronics in Agriculture, 142, 356368.

Wang, J., Duan, L., \& Yang, Y. (2018). An improvement crossover operation method in genetic algorithm and spatial optimization of heliostat field. Energy, 155, $15-28$.

Xiao, Y., Xie, Y., Kulturel-Konak, S., \& Konak, A. (2017). A problem evolution algorithm with linear programming for the dynamic facility layout problem-A general layout formulation. Computers \& Operations Research, 88, 187-207.

Yu, W., Li, B., Jia, H., Zhang, M., \& Wang, D. (2015). Application of multi-objective genetic algorithm to optimize energy efficiency and thermal comfort in building design. Energy and Buildings, 88, 135-143.

Zero, L., Bersani, C., Paolucci, M., \& Sacile, R. (2019). Two new approaches for the bi-objective shortest path with a fuzzy objective applied to HAZMAT transportation. Journal of hazardous materials, 375, 96-106.

Zhao, D., \& Liu, J. (2018). Study on network security situation awareness based on particle swarm optimization algorithm. Computers \& Industrial Engineering, 125, 764-775. 